\title{
Einflussfaktoren auf chronische Rückenschmerzen bei Pflegekräften in der Altenpflege in Rheinland-Pfalz
}

\section{Factors Influencing Chronic Back Pain in Care Workers Attending to The Elderly in Germany}

()(1) $\circledast \Theta$

\section{Autoren}

Dorothee Frey $^{1}{ }^{*}$, Sandra Rieger ${ }^{1}{ }^{*}$, Elisabeth Diehl ${ }^{1}$, Luis Carlos Escobar Pinzon ${ }^{1,2}$

Institute

1 Institut für Arbeits-, Sozial- und Umweltmedizin, Universitätsmedizin der Johannes Gutenberg-Universität Mainz, Mainz

2 Bundesanstalt für Arbeitsschutz und Arbeitsmedizin - BAuA, Berlin

\section{Schlüsselwörter}

chronische Rückenschmerzen, Lebenszeitprävalenz, Pflegekräfte, Altenpflege, Rheinland-Pfalz

\section{Key words}

chronic back pain, lifetime prevalence, care workers, elderly care, Germany

\section{Bibliografie}

DOI http://dx.doi.org/10.1055/s-0043-104693

Online-Publikation: 10.10 .2017

Gesundheitswesen 2018; 80: 172-175

(c) Georg Thieme Verlag KG Stuttgart · New York ISSN 0941-3790

\section{Korrespondenzadresse}

PD Dr. Luis Carlos Escobar Pinzon

Bundesanstalt für Arbeitsschutz und Arbeitsmedizin

Nöldnerstraße 40/42

10317 Berlin

escobarpinzon.luiscarlos@baua.bund.de

\section{ZUSAMMENFASSUNC}

Einleitung Die vorliegende Studie ermittelt die Lebenszeitprävalenz chronischer Rückenschmerzen bei Altenpflegekräften in rheinland-pfälzischen Einrichtungen und identifiziert potentielle Einflussfaktoren.

Methodik Im Rahmen einer Querschnittserhebung wurden 155 Pflegekräfte aus 5 Altenpflegeeinrichtungen untersucht und schriftlich befragt. Ausschnitte aus verschiedenen standardisierten Fragebögen wurden mit eigenen Fragen kombiniert und ergänzt durch eine körperliche Untersuchung mit 2 standardisierten Belastungstests. Die Daten wurden deskriptiv und bivariat sowie mittels binärlogistischer Regressionsanalyse ausgewertet.
Ergebnisse Die Pflegekräfte waren zu 83,8\% weiblich, das Durchschnittsalter lag bei 41 Jahren. Die Lebenszeitprävalenz chronischer Rückenschmerzen bei weiblichen Pflegekräften betrug 50,8\%, bei männlichen Pflegekräften lag sie bei 20,0\%. Als potentielle Einflussfaktoren für chronische Rückenschmerzen konnten ein schlechter subjektiver Gesundheitszustand und eine schwache Ausdauer der Rückenmuskulatur identifiziert werden.

Schlussfolgerung Die Lebenszeitprävalenz chronischer Rückenschmerzen war bei den Pflegekräften höher als in den Daten zur Allgemeinbevölkerung. Diskutierte Einflussfaktoren für Rückenschmerzen, wie z. B. zu viele patientenferne Aufgaben, Zeitdruck oder die Arbeit mit zu vielen Bewohnern erwiesen sich als nicht signifikant. Der subjektive Gesundheitszustand und die Ausdauer der Rückenmuskulatur standen dagegen in Zusammenhang mit Rückenschmerzen. Prospektive Studien zu physischen und psychischen Belastungen, Beanspruchungen und Ressourcen sind notwendig, um die Kausalität der hohen Lebenszeitprävalenz von chronischen Rückenschmerzen bei Altenpflegekräften besser verstehen zu können.

\section{ABSTRACT}

Introduction The present study determined the lifetime prevalence of chronic back pain in care workers attending to the needs of elderly in Rhineland-Palatinate facilities and identified potential influencing factors.

Methods In a cross-sectional survey, 155 care workers in 5 institutions for the elderly care were interviewed with a written questionnaire. Questions from different standardised questionnaires were combined with our own questions. We complemented the examination with 2 standardized exercise tests. The data were analysed using descriptive and bivariate as well as binary logistic regression analysis.

Results The nurses (83.8\%) were female, and the average age was 41 years. The lifetime prevalence of chronic back pain in female care workers was $50.8 \%$. In male elderly care workers this was $20.0 \%$. As potential influencing factors for chronic back pain, a bad subjective state of health and a weak endurance of back muscles could be identified. 
Conclusion The lifetime prevalence of chronic back pain was higher in the care workers taking care of the elderly than in the data of the general population. Discussed risk factors for back pain such as too many tasks not directly associated with caretaking, time pressure or too many elderly residents turned out as not significant. However, the subjective state of health and the endurance of back muscles were related to back pain. Prospective studies, which focus on physical and psychological stress, strain and resources, are needed to understand the causality of the high lifetime prevalence of chronic back pain in care workers in homes for the elderly better.

\section{Einleitung}

Die Lebenszeitprävalenz chronischer Rückenschmerzen unterscheidet sich in der deutschen Allgemeinbevölkerung nach Geschlecht, Alter und Bildungsstand [1,2].

Als Risikofaktoren für Rückenschmerzen gelten neben soziodemografischen Faktoren auch psychosoziale Faktoren, die für alle beruflich Tätigen [3] und speziell für Pflegekräfte nachgewiesen werden konnten [4]. Als berufsspezifische Ursachen für Rückenschmerzen werden u. a. der Transfer von Patienten (bspw. vom Bett zur Toilette) diskutiert [4].

Darüber hinaus wird in der bisherigen Literatur ein Zusammenhang zwischen der Rückenmuskulatur und Rückenschmerzen beschrieben, wobei sich eine schwache Ausbildung der Rückenmuskulatur als Risikofaktor für Rückenschmerzen herausstellte [5]. Neben den genannten Risikofaktoren spielen auch außerberufliche Faktoren eine Rolle, wie bspw. ein Partner bzw. eine weitere erwachsene Person im Haushalt [6].

Der Pflegeberuf ist international mit einer hohen Prävalenz von Rückenschmerzen assoziiert [6]. Daten zur Lebenszeitprävalenz chronischer Rückenschmerzen bei Pflegekräften sind den Autoren aus der nationalen Literatur nicht bekannt.

Ziel der vorliegenden Studie war es, die Lebenszeitprävalenz chronischer Rückenschmerzen bei Altenpflegekräften in RheinlandPfalz zu ermitteln und potentielle Einflussfaktoren zu identifizieren.

\section{Methodik}

\section{Studiendesign und Teilnehmer}

Im Rahmen der Querschnittstudie „Multifaktorielles Konzept zur Vermeidung muskuloskelettaler Beschwerden bei Pflegekräften in Rheinland-Pfalz“ wurden Daten zur Belastungs- und Gesundheitssituation von Altenpflegekräften in Rheinland-Pfalz erhoben.

Pflegekräfte aus 5 verschiedenen Einrichtungen der Altenpflege nahmen von April bis Mai 2012 freiwillig an einer ärztlichen Untersuchung sowie einer schriftlichen Befragung teil. Es gab keine Ein- oder Ausschlusskriterien.

\section{Definition Lebenszeitprävalenz chronischer Rücken- schmerzen}

Die Lebenszeitprävalenz chronischer Rückenschmerzen bezeichnet Rückenschmerzen, die mind. einmal im Leben über 3 Monate fast täglich aufgetreten sind [2]. Bei den Antworten der Befragten handelt es sich um eine subjektive Selbsteinschätzung.

\section{Ärztliche Untersuchung und Fragebogen}

Die ärztliche Untersuchung umfasste eine Allgemein- und Berufsanamnese sowie orthopädische Untersuchungen. Sie dauerte etwa
30 min. Im Rahmen der körperlichen Untersuchung wurden die Kraft der Bauchmuskulatur und die Ausdauer der Rückenmuskulatur erhoben. Die Ausdauer der Rückenmuskulatur wurde in 2 Kategorien (schwach/ stark) eingeteilt. Daneben wurden Merkmale der physischen Belastung (Heben/ Tragen von Lasten von $\geq 20 \mathrm{~kg}$ ) sowie die Dauer der Pflegetätigkeit (Jahre) erhoben.

Hauptthema der Befragung war die Prävalenz von chronischen Rückenschmerzen als primäres Outcome. Als sekundäre Outcomes wurden der Gesundheitszustand sowie arbeitsbedingte physische und psychische Belastungen und Beanspruchungen definiert. Angaben zum Gesundheitszustand wurden mittels Selbsteinschätzung im Fragebogen auf einer 5-stufigen Likert-Skala erhoben (sehr gut bis sehr schlecht).

Darüber hinaus beinhaltete der Fragebogen Teile des Nordischen Fragebogens zu muskuloskelettalen Beschwerden [7] und eigene Fragen zu beruflichen und privaten Rahmenbedingungen (u. a. Zusammenleben mit einem Partner im Haushalt). Des Weiteren wurden Teile des standardisierten BGWmiab-Fragebogens der Berufsgenossenschaft für Gesundheitsdienst und Wohlfahrtspflege (BGW) genutzt, um die berufsbezogene psychische Belastung (quantitative und qualitative Arbeitsbelastung, Arbeitsorganisation und soziales Umfeld) zu messen [8]. Aus den einzelnen BGWmiab-Items wurde anschließend die psychische Gesamtbelastung durch Addition der Mittelwerte aller Belastungsquellen errechnet. Je höher der erreichte Punktwert (Gesamtbelastung 1-20 bei 5 -stufiger Ratingskala 1 = nein gar nicht bis $5=$ ja genau), desto größer war die subjektiv empfundene psychische Arbeitsbelastung.

\section{Statistische Analyse}

Univariate Ergebnisse wurden mithilfe von gängigen deskriptivstatistischen Maßen dargestellt. Bivariate Analysen wurden für alle potentiellen Risikofaktoren durchgeführt. Anschließend wurde eine multiple Auswertung mittels binärer logistischer Regression (chronische Rückenschmerzen als abhängige Variable (nein/ ja) für alle signifikanten Variablen durchgeführt. Als Effektmaß wurden adjustierte relative Risiken (aOR) mit 95\%-Konfidenzintervallen berechnet (95\%-KI). Das Signifikanzniveau wurde auf $\alpha=0,05$ festgelegt. Die statistische Auswertung erfolgte mit SPSS ${ }^{\circledR} 21$ (IBM Corp. $@$, Armonk, NY, USA, 2012).

\section{Ergebnisse}

Die Größe der teilnehmenden Einrichtungen variierte zwischen 30 und 280 Betten (Mittelwert: 167). Insgesamt beschäftigten die Einrichtungen 470 Pflegekräfte (Mittelwert: 94; Range: 54-208). Von den 470 Pflegekräften erklärten sich 160 bereit an der Studie teilzunehmen (Teilnahmerate $34 \%$ ). 
- Tab. 1 Angaben zum Zusammenhang zwischen Rückenschmerzen und soziodemografischen Daten, beruflichen und außerberuflichen Rahmenbedingungen der Pflegekräfte $(n=155)$.

\begin{tabular}{|c|c|c|c|c|c|}
\hline \multirow[t]{2}{*}{ Variable } & \multirow[t]{2}{*}{ Kategorie } & \multirow[t]{2}{*}{$\mathbf{N}(\%)$} & \multicolumn{2}{|c|}{$\begin{array}{l}\text { Chronische Rückenschmerzen- } \\
\text { Lebenszeitprävalenz }\end{array}$} & \multirow[t]{2}{*}{ p-Wert } \\
\hline & & & $\mathrm{Ja}$ & Nein & \\
\hline & & & $N(\%)$ & $N(\%)$ & \\
\hline \multicolumn{6}{|l|}{ Soziodemografische Angaben } \\
\hline Alter & metrisch & & $\begin{array}{c}\text { Median } 49 \\
(50,5 \%)\end{array}$ & $\begin{array}{c}\text { Median } \\
36,5(49,5 \%)\end{array}$ & $<0,001^{\text {a }}$ \\
\hline \multirow[t]{2}{*}{ Geschlecht } & weiblich & $130(83,8)$ & $66(50,8)$ & $64(49,2)$ & $0,005^{b}$ \\
\hline & männlich & $25(16,1)$ & $5(20,0)$ & $20(80,0)$ & \\
\hline \multirow[t]{3}{*}{ Höchster Bildungsabschluss } & Hauptschulabschluss & $56(36,1)$ & $34(60,7)$ & $22(39,3)$ & $0,014^{b}$ \\
\hline & Mittlere Reife/ POS & $53(34,2)$ & $22(41,5)$ & $31(58,5)$ & \\
\hline & Fachabitur/ Abitur & $46(29,7)$ & $15(32,6)$ & $31(67,4)$ & \\
\hline \multicolumn{6}{|c|}{ Außerberufl. Rahmenbedingungen } \\
\hline \multirow[t]{2}{*}{ Partner im Haushalt ${ }^{\mathrm{d}}$} & nein & $45(29,4)$ & $14(31,1)$ & $31(68,9)$ & $0,032^{b}$ \\
\hline & ja & $108(70,6)$ & $55(50,9)$ & $53(49,1)$ & \\
\hline \multirow[t]{2}{*}{ Gesundheitszustand } & schlecht/ mittelmäßig & $59(38,1)$ & $43(72,9)$ & $16(27,1)$ & $<0,001^{b}$ \\
\hline & gut/ sehr gut & $96(61,9)$ & $28(29,2)$ & $68(70,8)$ & \\
\hline \multirow[t]{2}{*}{ Rückenmuskulaturc } & schwach & $57(38)$ & $38(67,7)$ & $19(33,3)$ & $<0,001^{b}$ \\
\hline & stark & $93(62)$ & $31(33,3)$ & $62(67,7)$ & \\
\hline \multicolumn{6}{|c|}{ Berufliche Rahmenbedingungen } \\
\hline \multirow[t]{3}{*}{ mind. $20 \mathrm{~kg}$ Heben/ Tragen ${ }^{\mathrm{d}}$} & nein & $15(9,8)$ & $3(20,0)$ & $12(80,0)$ & $<0,001^{b}$ \\
\hline & ja, bis 10 Jahre & $62(40,5)$ & $18(29,0)$ & $44(71,0)$ & \\
\hline & ja, über 10 Jahre & $76(49,7)$ & $48(63,2)$ & $28(36,8)$ & \\
\hline Berufsjahre im Pflegeberuf & metrisch & Median 13,3 (100) & Median 20,7 $(56,0)$ & Median 9,9 $(44,0)$ & $<0,001^{\text {a }}$ \\
\hline Psychische Gesamtbelastunge & metrisch & Median $12(100)$ & Median $12,3(48,4)$ & Median 11,7 $(51,6)$ & $0,017^{a}$ \\
\hline
\end{tabular}

5 Personen mussten von den körperlichen Belastungstests ausgeschlossen werden, womit 155 Teilnehmer in den folgenden Analysen berücksichtigt werden konnten.

Die Pflegekräfte waren zu 83,8\% weiblich und im Durchschnitt 41 Jahre alt (Median: 44,0; SD:13,0; Range: 17-62). Die Lebenszeitprävalenz von chronischen Rückenschmerzen betrug 45,8\%. Stratifiziert nach Geschlecht lag sie für Frauen bei 50,8\% und bei Männern bei 20,0\% ( $\triangleright$ Tab. 1).

\section{Bivariate Analyse}

Der Zusammenhang zwischen möglichen Einflussfaktoren und chronischen Rückenschmerzen kann der \ Tab. 1 entnommen werden. Pflegende mit chronischen Rückenschmerzen waren signifikant älter als Pflegende ohne Rückenschmerzen ( $p<0,001)$. Weibliche Pflegekräfte $(p=0,005)$ und Pflegekräfte mit Hauptschulabschluss gaben häufiger Rückenschmerzen an, als Pflegekräfte mit einem höheren Schulabschluss ( $p=0,014)$. Pflegekräfte mit einem guten bzw. sehr guten Gesundheitszustand und Pflegekräfte mit einer starken Rückenmuskulatur litten seltener an Rückenschmerzen $(p<0,001)$. Pflegekräfte mit chronischen Rückenschmerzen gaben eine stärkere psychische Gesamtbelastung $(p=0,017)$ an. Pflegekräfte ohne Partner im Haushalt hatten seltener Rückenschmerzen ( $p=0,032)$. Pflegekräfte, die seit mehr als 10 Jahren häufig mehr als $20 \mathrm{~kg}$ Lastenhandhabung angaben, hatten häufi- ger Rückenschmerzen und waren doppelt so lange im Pflegeberuf tätig (jeweils $p<0,001$ ). Aufgrund der starken Korrelation (Spearman Rho $=0,68, p<0,001$ ) zwischen den zuletzt genannten Variablen wurde nur letztere in die multiple Analyse aufgenommen.

\section{Multiple Zusammenhangsanalyse}

Für 2 Einflussfaktoren konnte in der multiplen Analyse ein erhöhtes Risiko für chronische Rückenschmerzen festgestellt werden: Pflegekräfte mit einem schlechten bzw. mittelmäßigen Gesundheitszustand hatten ein 3,56-fach höheres Risiko als Pflegekräfte mit einem guten bzw. sehr guten Gesundheitszustand ( $p=0,004)$. Pflegekräfte mit einer schwachen Rückenmuskulatur wiesen ein 3,94-fach höheres Risiko auf ( $p=0,004)$ als Pflegekräfte mit einer starken Rückenmuskulatur. Die weiteren Einflussfaktoren psychische Belastung Partner im Haushalt, Alter, Geschlecht, Schulabschluss und Berufsjahre erwiesen sich im Regressionsmodell als nicht signifikant.

\section{Diskussion}

Die Lebenszeitprävalenz von chronischen Rückenschmerzen betrug 45,8\% und liegt damit deutlich über der Lebenszeitprävalenz der deutschen Allgemeinbevölkerung von 27,3\% [1]. Die Ergebnisse decken sich mit der internationalen Literatur, die ebenfalls über 
höhere Prävalenzen von chronischen Rückenschmerzen bei Personen in Pflegeberufen berichtet [6]. Zugleich stärkt die vorliegende Studie die bisher schwache nationale Datenlage zur Thematik. Weiterhin decken sich die Ergebnisse zu Geschlecht, Schulbildung und Alter mit der vorhandenen Literatur [1, 2, 9].

\section{Subjektiver Gesundheitszustand}

In der vorliegenden Studie war das statistische Risiko chronische Rückenschmerzen zu erleiden für Pflegekräfte mit einem schlechten bzw. mittelmäßigen allgemeinen Gesundheitszustand erhöht. Ähnliche Ergebnisse finden sich auch in der wissenschaftlichen Literatur [10].

\section{Ausdauer der Rückenmuskulatur}

Eine starke Ausdauer der Rückenmuskulatur reduziert das Risiko an chronischen Rückenschmerzen zu erkranken. Die Bedeutung der Rückenmuskulatur bei der Entstehung und Aufrechterhaltung von chronischen Rückenschmerzen wird auch in der wissenschaftlichen Literatur diskutiert [5].

\section{Limitationen}

Die Ergebnisse der vorliegenden Studie sind einigen wesentlichen Limitationen unterworfen: einem kleinen Kollektiv an teilnehmenden Pflegekräften, einem Selektions- und Informationsbias durch die Freiwilligkeit der Teilnahme der Einrichtungen, einer möglichen erhöhten Teilnahmequote durch Rückenschmerzbetroffene und einer potentiellen Unterschätzung des Parameters Zeitmangel. Das Querschnittsdesign lässt darüber hinaus keine Aussagen zur (reversen) Kausalität zu, da keine reliablen Daten zur zeitlichen Abfolge verfügbar sind. Die identifizierten Zusammenhänge können daher nur Hinweise auf potentielle Einflussfaktoren liefern.

\section{Schlussfolgerungen}

Chronische Rückenschmerzen stellen ein ernstzunehmendes Problem bei Altenpflegekräften dar. Präventionsprogramme sollen die potentiellen Risikofaktoren subjektiver Gesundheitszustand und Ausdauer der Rückenmuskulatur besonders berücksichtigen. Die Daten sollten in einem größeren Kollektiv in prospektiven Studien überprüft werden, um kausale Rückschlüsse ziehen zu können. Zudem können nur in einem größeren Kollektiv mögliche Präventionsansätze differenziert für die unterschiedlichen Bedürfnisse und Zielgruppen entwickelt und erprobt werden.

\section{Einhaltung ethischer Richtlinie}

Positive Bewertung der Studie durch die Ethikkommission der Landesärztekammer Rheinland-Pfalz.

\section{Danksagung}

Wir danken unseren Förderern und Kooperationspartnern: Unfallkasse Rheinland-Pfalz, Berufsgenossenschaft für Gesundheitsdienst und Wohlfahrtspflege, - BGW. Europäischer Sozialfonds Rheinland-Pfalz und dem Ministerium für Soziales, Arbeit, Gesundheit und Demografie Rheinland-Pfalz.

Interessenkonflikt

Die Autoren geben an, dass kein Interessenkonflikt besteht.

Literatur

[1] Neuhauser H, Ellert U, Ziese T. Chronische Rückenschmerzen in der Allgemeinbevölkerung in Deutschland 2002/2003: Prävalenz und besonders betroffene Bevölkerungsgruppen. Gesundheitswesen 2005; 67: 685-693

[2] Raspe H. Rückenschmerzen. Gesundheitsberichterstattung des Bundes, Band 53. Berlin: Robert-Koch-Institut; 2012

[3] Stadler P, Spieß E. Arbeit-Psyche-Rückenschmerz: Einflussfaktoren und Präventionsmöglichkeiten. Arbeitsmed. Sozialmed. Umweltmed 2009; 44: 68-76

[4] Simon M, Tackenberg P, Nienhaus A et al. Back or neck-pain-related disability of nursing staff in hospitals, nursing homes and home care in seven countries - results from the European NEXT-Study. Int J Nurs Stud 2008; 45: 24-34

[5] Luoto S, Heliövaara M, Hurri $\mathrm{H}$ et al. Static back endurance and the risk of low-back pain. Clin biomech (Bristol, Avon) 1995; 10: 323-324

[6] Smith BH, Elliott AM, Hannaford PC et al. Factors related to the onset and persistence of chronic back pain in the community: results from a general population follow-up study. Spine 2004; 29: 1032-1040

[7] Caffier G, Steinberg U, Liebers F. Praxisorientiertes Methodeninventar zur Belastungs- und Beanspruchungsbeurteilung im Zusammenhang mit arbeitsbedingten Muskel-Skelett-Erkrankungen. Schriftenreihe der Bundesanstalt für Arbeitsschutz und Arbeitsmedizin (BAuA), Forschung Fb 850. Dortmund/Berlin: BAuA; 1999

[8] Berufsgenossenschaft für Gesundheitsdienst und Wohlfahrtspflege (BGW). Mitarbeiterbefragung Psychische Belastung und Beanspruchung. BGWmiab für die Pflege und den stationären Wohnbereich der Behindertenhilfe. Hamburg: BGW; 2011

[9] Kromark K, Dulon M, Nienhaus A. Health indicators and preventive behaviour of older employees in geriatric care. Gesundheitswesen 2008; 70: 137-144

[10] Harcombe H, McBride D, Derrett S et al. Physical and psychosocial risk factors for musculoskeletal disorders in New Zealand nurses, postal workers and office workers. Injury prevention. Journal of the International Society for Child and Adolescent Injury Prevention 2010; 16: $96-100$ 